\title{
Robot-assisted task-oriented upper extremity skill training in cervical spinal cord injury: a feasibility study
}

\author{
DAMM Vanmulken ${ }^{1,2}$, AIF Spooren ${ }^{2}$, HMH Bongers $^{1}$ and HAM Seelen ${ }^{2,3}$
}

Study design: Prospective multiple case study.

Objectives: To test (1) the feasibility of haptic robot technology (Haptic Master (HM)) use to improve arm-hand function (AHF) and arm-hand skill performance (AHSP) in persons with a cervical spinal cord injury (C-SCI), (2) inventory participants' motivation and expectation to work with the robot technology used and (3) to descriptively report the results in individual cases.

Setting: Rehabilitation Centre.

Methods: Five C-SCl patients were trained for 6 weeks, 3 days per week, 60 min per day. Therapists filled out the Usefulness, Satisfaction and Ease-of-use questionnaire (USE). The Intrinsic Motivational Inventory (IMI) and credibility and expectancy questionnaire (CEQ) were filled out by participants. Performance at activity level was gauged using the Van Lieshout test for AHF in Tetraplegia and the Spinal Cord Independence Measure. Function level was gauged using muscle strength testing and the International Classification for Surgery of the Hand in Tetraplegia.

Results: As to the feasibility of the application of haptic robot technology, the mean USE score was 65\%. Mean IMI and CEQ results were $67 \%$ and $60 \%$, respectively. Participants were motivated to train with the HM. All participants rated credibility higher than expectations regarding the improvement. In the current patients, little progress was demonstrated at the International Classification of Functioning, Disability and Health function and the activity level.

Conclusion: It is feasible to train $\mathrm{C}-\mathrm{SCl}$ persons with the HM. Therapists report that working with the HM is easy to learn and easy to perform. Usability of the HM may be improved. Further research is needed to assess in which group of $\mathrm{C}-\mathrm{SCl}$ and at which stage of rehabilitation $\mathrm{HM}$ training may be most beneficial.

Spinal Cord (2015) 53, 547-551; doi:10.1038/sc.2014.250; published online 3 February 2015

\section{INTRODUCTION}

Persons with a cervical spinal cord injury (C-SCI) have to cope with a limited arm-hand function (AHF), which causes difficulties or even an inability to perform daily activities. ${ }^{1}$ In the present study, AHF refers to the International Classification of Functioning, Disability and Health function level, whereas arm-hand skill performance (AHSP) refers to the International Classification of Functioning, Disability and Health activity level and includes a broad range of activities of different complexity. ${ }^{2}$

Patients with a C-SCI give a high priority to therapy aimed at improving their AHSP, relative to other therapy aims in rehabilitation, because it may strongly improve their quality of life. ${ }^{3}$ As rehabilitation time is too short to train a comprehensive set of skills, new therapy approaches need to be developed. These new approaches should take into account the client-dependent versatility of training goals, the limited amount of time available, the necessary prerequisites as to training physiology (like repetition maximum, dynamic and endurance strength training, coordination, overload, training specificity and functional training ${ }^{4}$ ) and motor learning (like context specificity, distribution-based practice, whole learning, shaping, feedback, guidance and variability), ${ }^{5}$ as well as patients' motivation and the (concomitant) level of challenge to the patient. To this end, Spooren et al. ${ }^{6}$ developed, evaluated and implemented a modular task-oriented client-centred training programme to improve AHSP (ToCUEST). In clinical rehabilitation practice, however, it is well known that high levels of movement repetition and lack of training variation may lead to patients becoming bored, and thus may lead to decreased motivation to train and to lower levels of therapy adherence. To explore the possibilities for extending ToCUEST's training variation, which, in turn, might further improve patients' training motivation, 5,7 the use of training technology was considered, analogous to the work of Timmermans et al., ${ }^{8,9}$ who developed and evaluated a technologyassisted task-oriented arm training approach (T-TOAT) for persons with stroke. The use of such a training approach to improve AHSP in persons with C-SCI has not yet been reported. This type of training may improve patients' engagement, because it provides more variation in their (regular) therapy programme. Also, patients are enabled to perform activities they cannot perform without the (arm)support of the haptic robot. This, in turn, increases motivation.

The main aim of this study has been to test the feasibility of the use of haptic robot technology to improve the training content variability regarding the AHSP training. As part of this study, participants'

\footnotetext{
${ }^{1}$ Adelante Rehabilitation Centre, Hoensbroek, The Netherlands; ${ }^{2}$ Spinal Cord Injury Department, Adelante Centre of Expertise in Rehabilitation and Audiology, Hoensbroek, The Netherlands and ${ }^{3}$ Department of Rehabilitation Medicine, Research School CAPHRI, Maastricht University, Maastricht, The Netherlands

Correspondence: DAMM Vanmulken, Spinal Cord Injury Department, Adelante Centre of Expertise in Rehabilitation and Audiology, Zandbergsweg 111,6432 CC Hoensbroek, The Netherlands.

E-mail: d.vanmulken@adelante-zorggroep.nl

Received 9 April 2014; revised 24 December 2014; accepted 29 December 2014; published online 3 February 2015
} 
motivation and expectation as to the technique used were also inventoried. Changes on individuals' function and activity level are briefly reported.

\section{MATERIALS AND METHODS}

\section{Participants}

Participants were recruited from the databases of Adelante Rehabilitation Centre. Inclusion criteria were as follows: C-SCI level C4-C7; classified as A-D on the ASIA Impairment Scale; ${ }^{10}$ age between 18 and 70 years; postrehabilitation phase $>1$ year; problems with AHSP; minimal wrist extension of $10^{\circ}$; maximal finger extension of $30^{\circ}$; active in daily life; and able to sit up for at least $4 \mathrm{~h}$. Exclusion criteria were as follows: additional neurologic, orthopaedic or rheumatic illnesses, which may interfere with AHF; severe spasticity (Ashworth score $\geqslant 3$ ); and inability to perform AHF and AHSP measurements.

\section{Design}

This descriptive study included several cases of individual C-SCI patients. Usability, from the perspective of the therapists who conducted the training, was measured at the end of the training phase. Patient's motivation and expectations were inventoried after the first week of the training. The participants were trained three times per week for 6 weeks. Measurements regarding the function level and the activity level were performed 1 week before the training started (T0), after 4 weeks of training (T1) and 1 week after the cessation of training (T2). Each training day consisted of $2 \times 30 \mathrm{~min}$, interspaced by a 30 -min break.

\section{Materials and intervention}

Trained skills were, among others, 'eating with fork and knife', 'taking money out of a purse' and 'moving a cup'. Per participant client-specific skills were chosen, in accordance with their level of impairment. All skills were broken down into subskills or components. ${ }^{9}{ }^{11}$ For each of these components, exercises were offered at increasing levels of difficulty with the feedback delivered according to the shaping principles. ${ }^{12}$ Exercise progression was based on principles of training physiology ${ }^{4}$ and motor learning. ${ }^{5}$ Regarding training physiology, a goal-dependent functional and specific skill training was offered with training loads exceeding the patient's metabolic capacity (overload principle $^{4}$ ). As to motor learning principles, 'over-learning strategies' (that is, continued practice after performance), 'exercise variability' to improve patient's motivation and conserve training effect and 'guidance and feedback ${ }^{5,9}$ (provided by the haptic robot device ${ }^{8,11}$ ) were used. Patients were offered a task-oriented training, that is, a repetitive training of self-selected, real life activities, to also improve their motivation. ${ }^{11}$ This training was supported by haptic robot technology (Haptic Master (HM), Moog, Nieuw-Vennep, The Netherlands) (see Figures 1 and 2).

The HM is three degrees of freedom admittance-controlled robotic system. It is suitable for larger workspaces and high forces can be exerted. The patient's arm was attached to the robot using a customised gimbal and an orthosis at the

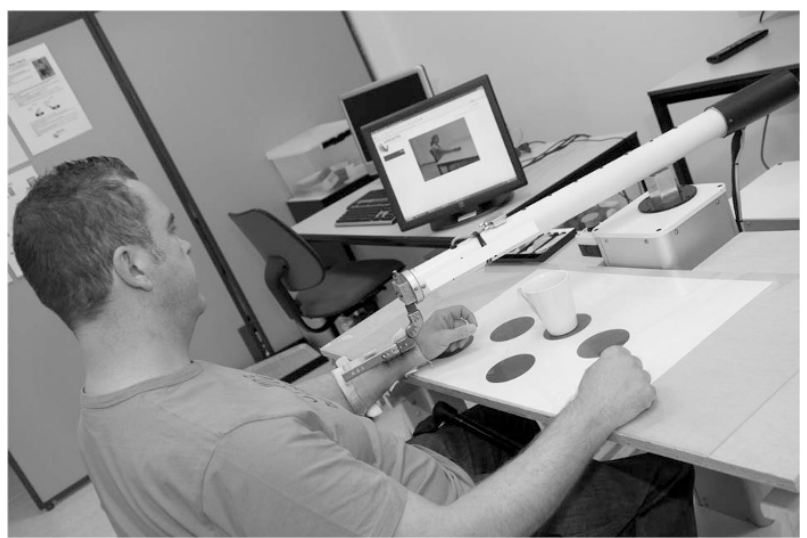

Figure 1 Move a cup with support of the Haptic Master. distal part of the forearm. Dedicated software, called 'Haptic-TOAT', as used in the T-TOAT study of Timmermans et al., ${ }^{11}$ was applied. The first time the patient performed a specific arm-hand skill, the spatial movement trajectory of the orthosis was electronically recorded by the HM in terms of 3D positions (that is, $x, y, z$ coordinates) with a sample rate of $100 \mathrm{~Hz}$. During this recording, the patient's arm, which was attached to the HM end-effector, was moved along the optimal path either passively (by the therapist) or assisted actively (by the patient himself, aided by the therapist). If necessary the movement path recorded could be edited (adapted) or filtered (smoothed) by the Haptic-TOAT software.

The HM assisted in supporting the patient's arm movements during skill performance. It allowed patients to practise in three modes: the passive mode in which the patient's arm was moved by the device; the active-assisted mode in which the patient was partially assisted in performing a specific movement or task; and the active mode in which the patient performed the required movement or task against moderate resistance. The training activities were instructed using a video. Training was supervised by senior occupational therapists. During training the HM provided kinaesthetic feedback regarding the movement performed. For example, if the patient's movement deviated too much from the predefined (that is, recorded) movement trajectory, the HM led the arm back onto the correct path using spring-like forces. Another example of the HM features was 'tunnelling', in which the predefined movement trajectory was modelled as a 3D tunnel beyond which no movement was possible, thus preventing the excessive movement deviations. It was possible for patients to train on bimanual activities. However, only one arm was supported by the HM. Prior to the start of the training patients were allowed to choose which arm was to be trained using the HM. By training bimanual tasks the non-supported arm was also trained.

The Medical Ethics Committee of Maastricht University approved the research protocol. All participants gave their written informed consent before the start of the study.

\section{Measurements}

All measurements were conducted by experienced therapists.

Usability. The Usefulness, Satisfaction and Ease-of-use questionnaire ${ }^{13}$ was filled out by the occupational therapists to gauge the feasibility of the HM training and to gain insight in its usability and ease of use. It consists of four items (that is, 'usefulness', 'ease of use', 'easy to learn' and 'satisfaction'), each containing between 4 and 11 subitems, all rated on a 7-point Likert scale.

Motivation and expectations. The intrinsic motivation inventory (IMI) ${ }^{14}$ was used to assess participants' subjective experience related to intrinsic motivation and self-regulation. It consists of six different items (that is, 'interest/pleasure', 'experienced competence', 'effort/importance', 'pressure/tension', 'value/benefit' and 'solidarity'). The maximum total IMI score is 42 . Both reliability and validity of the IMI have been established in various populations. ${ }^{14}$

The credibility/expectancy questionnaire ${ }^{15}$ assesses the credibility and participant's expectations regarding training. Its total score ranges between 3 and 27. The questionnaire has a high internal consistency within each factor and a good test-retest reliability. ${ }^{15}$

Activity level. The Van Lieshout test for Tetraplegia-short form ${ }^{16,17}$ was used to asses actual performance of arm-hand skills. Its 10 items each range between 0 and 5. The Van Lieshout test for Tetraplegia-short form is responsive to measure changes in AHSP during rehabilitation in people with a C-SCI. ${ }^{17}$

The Spinal Cord Independence Measure (version III) ${ }^{18}$ rates selfcare, respiration and sphincter management, mobility in the room and toilet and mobility indoors and outdoors (on even surface). It is reliable and valid for traumatic spinal cord injury. ${ }^{18}$ The maximum score is 100 .

Function level. The International Classification for Surgery of the Hand in Tetraplegia ${ }^{19}$ and the MicroFet_2 (Hoggan Health Industries, Salt Lake City, UT, USA) strength measurement were used. ${ }^{20}$ The International Classification for Surgery of the Hand in Tetraplegia examines the motor and sensory function and classifies the neurological status. The maximum score 9 indicates only loss of intrinsic hand-muscle function, whereas score 0 indicates there are 
no muscles suitable for surgical transfers. The MicroFet measures force $(N)$ exerted. Each measurement is performed three times, the highest of which is then used.

\section{Data analysis}

As this was a feasibility study; all data are reported descriptively.

\section{RESULTS}

Participants' characteristics are presented in Table 1.

P2 was classified as an ASIA Impairment Scale-A patient. It should be noted, however, that there was a zone of partial innervation. ${ }^{10}$ (Details: see Table 1.) P2 had one actively functioning hand, whereas the other hand was used with a tenodesis grip. P5 underwent reconstructive arm-hand surgery. He had a triceps reconstruction and surgery for wrist extension. He used both the hands with a tenodesis function.

Three participants completed the whole training programme. Early withdrawal of 2 patients was due to health issues unrelated to the training. Four persons chose to train the side with the poorest hand function. P3 trained the left hand, because the right hand did not have enough active function to be trained with HM. P3 was, however, able to use the right hand for support in bimanual tasks. No adverse training effects were found. All 5 participants were able to train with the HM.

In Table 2, an overview of the baseline level of functioning of the three participants who completed the whole training is presented.

P4 had already high baseline levels. He was ADL (activities of daily living) independent and used a manual wheelchair. P2 also used a

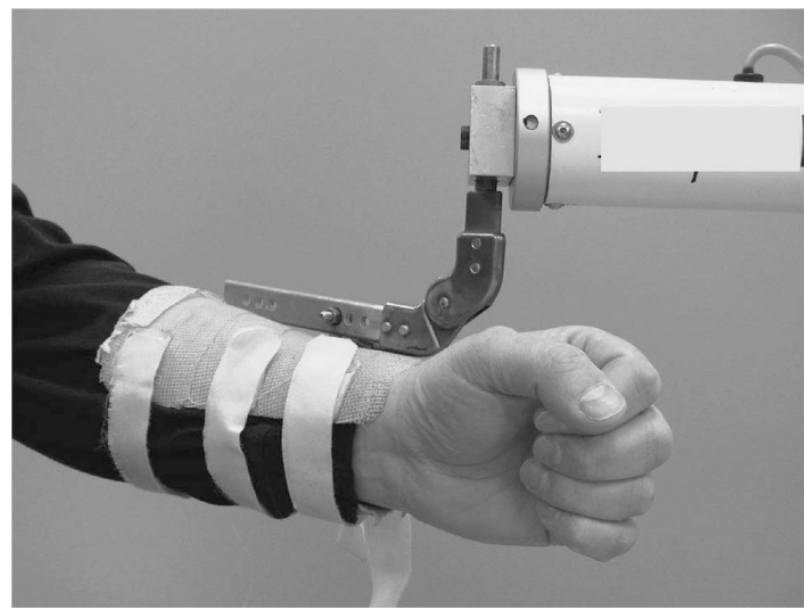

Figure 2 Orthosis attached to the HM. manual wheelchair but needed some assistance for movements outside and for ADL care. P5 used an electric wheelchair and was for the greater part $\mathrm{ADL}$ dependent.

The average (normalised) Usefulness, Satisfaction and Ease-of-use questionnaire results, rated by the therapists, amounted to $71.0 \%$ (s.d.: $13.4 \%$ ) for the item 'ease of use', $93.9 \%$ (s.d.: $7.5 \%$ ) for the item 'easy to learn', $59.2 \%$ (s.d.: $25.5 \%$ ) for 'satisfaction' and $47.0 \%$ (s.d.: $18.4 \%$ ) for 'usefulness'. The average (normalised) total score of the Usefulness, Satisfaction and Ease-of-use questionnaire corresponded to $65.1 \%$ (s.d.: $5.0 \%$ ).

As to the IMI, all participants scored low on 'pressure/tension' (mean normalised score: $21.1 \%$, s.d.: $1.3 \%$ ). The mean (normalised) score of the item 'effort/importance' was highest-that is, $80.6 \%$ (s.d.: 1.8\%). The items 'interest/pleasure', 'experienced competence' and 'value/benefit' all were rated above average-that is, $73.5 \%$ (s.d.: 3.2\%), 72.4\% (s.d.: 4.1\%), 72.7\% (s.d.: 4.7\%) and 69.7\% (s.d.: 4.4\%), respectively. P1 and P2 had the largest motivation, whereas P5 was least intrinsically motivated. The mean IMI score of the whole group was $66.1 \%$ (s.d.: $14.7 \%$ ).

Four participants scored high on credibility - that is, normalised credibility scores $\geqslant 74 \%$. In each individual participant, the credibility scores were higher than the expectancy scores. The mean

\section{Table 2 Level of functioning at baseline}

\begin{tabular}{lccc}
\hline Trained side & P2 Left & P4 Right & P5 Right \\
\hline MRC (grades: 0-5) & & & \\
Elbow flexion & 5 & 5 & 4 \\
Elbow extension & 4 & 5 & 3 \\
Wrist extension & 4 & 4 & 4 \\
Finger flexion & 4 & 3 & 0 \\
Finger abduction & 2 & 2 & 0 \\
& & & \\
Muscle strength (N) & $155 / 66$ & $226 / 86$ & $144 / 48$ \\
Elbow flexion/extension ${ }^{\text {b }}$ & $57 / 117 / 88$ & $124 / 280 / 186$ & $46 / 84 / 160$ \\
Shoulder exo/endo/abd & 62 & 188 & 44 \\
Wrist extension & 3 & 5 & 1 \\
ICSHT & 29 & 48 & 17 \\
VLT-sf (max=50) & 51 & 72 & 36 \\
SCIM (max=100) & & & \\
\hline
\end{tabular}

Abbreviations: abd, abduction; endo, endorotation; exo, exorotation; ICSHT, International Classification for Surgery of the Hand in Tetraplegia; $P$, participant; MRC, Medical Research Council; SCIM, spinal cord independence measure; VLT-sf, Van Lieshout Hand function test for Tetraplegia (short form).

aP5 had triceps reconstruction and surgery for wrist extension. He had a tenodesis grip. ${ }^{b}$ All muscle-strength tests were performed while the participants were seated. When elbow extensor and flexor muscle strength was tested, the upper arm was held against the trunk, that is, in a $0^{\circ}$ shoulder flexion position, whereas the forearm was supinated with an elbow flexion angle of $90^{\circ}$. Muscle strength of the elbow extensors was measured in the vertical direction, directed with gravity.

Table 1 Participant characteristics

\begin{tabular}{|c|c|c|c|c|c|c|c|}
\hline Patient code & Gender (M/F) & Age (years) & Postinjury time (years) & ASIA score & AIS score & Hand dominance (L/R) & Trained hand $(L / R)$ \\
\hline $\mathrm{P} 1^{\mathrm{a}}$ & $\mathrm{F}$ & 70 & 2.5 & C6 & $\mathrm{D}$ & $\mathrm{R}$ & $\mathrm{L}$ \\
\hline $\mathrm{P} 2^{\mathrm{b}}$ & M & 25 & 9.0 & C7 & $A$ & $\mathrm{R}$ & $\mathrm{L}$ \\
\hline P3a & M & 62 & 10.5 & $\mathrm{C} 5-\mathrm{C} 7$ & $\mathrm{C}$ & $\mathrm{L}$ & $\mathrm{L}$ \\
\hline P4 & M & 34 & 3.5 & C7 & B & $\mathrm{R}$ & $\mathrm{R}$ \\
\hline P5 & M & 45 & 15.5 & C5 & B & $\mathrm{R}$ & $\mathrm{R}$ \\
\hline
\end{tabular}

Abbreviations: AIS, ASIA Impairment Scale; $C$, cervical level; $F$, female; $L$, left; $M$, male; $P$, participant; $R$, right; $T$, Thoracic level.

aparticipants who did not complete the whole programme.

bZone of partial innervation: sensory right: T11, left T11; motor right: T1, left: T1. 
(normalised) total score on the credibility/expectancy questionnaire was $60.7 \%$ (s.d.: $20.6 \%$ ).

No large improvements at activity level were found. As to the Spinal Cord Independence Measure, all participants scored high on 'self care' and 'respiration and sphincter management', whereas they scored low on 'mobility'. There were no discernable differences on the spinal cord independence measure between the different times of measurement.

Between T1 and T2 participant P2 and P5 progressed on one Van Lieshout test for Tetraplegia-short form item. P4 already had a maximum score of 5 on all the tasks, except for 'thumb grip' and 'pen grip' (score 4).

The International Classification for Surgery of the Hand in Tetraplegia did not change in any of the participants. As to the muscle strength, P2 improved on all major arm muscles (improvement ranging between $8 \%$ and 22\%). P5 improved on elbow flexion (23\%), shoulder abduction (13\%) and wrist extension (17\%). $\mathrm{P} 4$ showed no progress on muscle strength.

\section{DISCUSSION}

This study aimed to investigate the feasibility to train AHSP in persons with a C-SCI using robot technology. Five C-SCI patients with different lesion levels and different levels of AHF were trained with the HM. Usefulness, Satisfaction and Ease-of-use questionnaire data revealed that working with the robotic device was easy to learn and easy to do. The usability of the current robotic system may, however, be improved. Currently, it is only possible to train arm activities and skills within a limited movement space. Participants reported that the orthosis that attaches the arm to the HM sometimes limits fluent movement.

Therapists applied the forearm orthosis, which was considered very easy to do. One participant was even able to apply the orthosis himself. The robotic system facilitated training of different activities and skills, at variable levels of task difficulty. Even bimanual skills could be trained on. However, because of current software and hardware limitations, complex movements in a larger movement space and with many different movement components were not (yet) possible. With the current software a therapist's intervention is needed to start each different activity separately. Being able to programme sequences of activities may enable patients to train more independently.

The scores on intrinsic motivation, credibility and expectancy were diverse among the participants. The least motivated participant also had the lowest expectations regarding improvement. This participant had the highest lesion level (C5, ASIA Impairment Scale-B) and the longest time since injury. Another participant, with a lower than average motivation and expectancy score, already had a rather good AHF, which may have been the reason for his lower scores. Since time post injury was long, participants did not expect large improvements regarding their arm-hand function as a result of this training. Participants did, however, report that, in their own opinion, the robotic system may be an adequate tool to train AHSP in an earlier phase post injury. Maybe there is a specific subgroup of C-SCI patients, that is, those with a relatively short post-injury time and a moderately to severely impaired arm-hand function, who may be highly motivated and may optimally benefit from robot-assisted training. This, however, needs further investigation.

\section{Considerations and future research}

In this feasibility study, participants were trained in an exercise lab at the rehabilitation centre. The (expensive) robotic device cannot be easily transported to a patient's room or home, which limits its widespread use. Also, the effect of intensity, frequency and duration of the training programme on possible improvement of AHF and AHSP needs to be further investigated in a broader group of persons with a C-SCI. For example, future studies should investigate whether effects of training with technology regarding AHF and AHSP differ between C-SCI patients in the chronic phase and the acute phase. Furthermore, it is important to investigate the possible differences in training with technology among persons with a higher cervical lesion (C4-C5) with poorer AHF and AHSP, results of which may then be compared with those of persons with a lower cervical lesion (C6-C8).

\section{CONCLUSION}

It is feasible to train persons with a C-SCI with robotic systems like the HM. Working with the HM is easy to learn and easy to do, although the usability of this robotic system may be improved. Participants found the training to be credible. Most of them were motivated, although expectations as to improvement on AHF and AHSP were lower. The study population was not large enough to ascertain whether training with the HM may improve AHSP in persons with a C-SCI who finished active rehabilitation 1 year earlier.

\section{DATA ARCHIVING}

There were no data to deposit.

\section{CONFLICT OF INTEREST}

The authors declare no conflict of interest.

1 Binder H. Traumatic spinal cord injury. Barnes MP, Good DC. Neurological Rehabilitation. Elsevier BV: Edinburgh, 2013; 411-426.

2 Spooren AI, Janssen-Potten YJ, Snoek GJ, Ijzerman MJ, Kerckhofs E, Seelen HA Rehabilitation outcome of upper extremity skilled performance in persons with cervical spinal cord injuries. J Rehabil Med 2008; 40: 637-644.

3 Snoek GJ, IJzerman MJ, Hermens HJ, Maxwell D, Biering-Sorensen F. Survey of the needs of patients with spinal cord injury: impact and priority for improvement in hand function in tetraplegics. Spinal Cord 2004; 42: 526-532.

4 McArdle WD, Katch FI, Katch VL. Exercise Physiology: Nutrition, Energy, and Human Performance. Lippincott Williams \& Wilkins: Philadelphia, PA, 2010.

5 Magill RA. Motor Learning and Control. Concepts and applications. McGraw-Hill Companies: New York. 2011

6 Spooren Al, Janssen-Potten YJ, Kerckhofs E, Bongers HM, Seelen HA. ToCUEST: a task-oriented client-centered training module to improve upper extremity skilled performance in cervical spinal cord-injured persons. Spinal Cord 2011; 49: 1042-1048.

7 Popovic MD, Kostic MD, Rodic SZ, Konstantinovic LM. Feedback-mediated upper extremities exercise: increasing patient motivation in poststroke rehabilitation. Biomed Res Int 2014, 520374.

8 Timmermans AA, Lemmens RJ, Monfrance M, Geers RP, Bakx W, Smeets RJ et al. Effects of task-oriented robot training on arm function, activity, and quality of life in chronic stroke patients: a randomized controlled trial. J Neuroeng Rehabil 2014; 11 : 45

9 Timmermans AA, Seelen HA, Willmann RD, Kingma H. Technology-assisted training of arm-hand skills in stroke: concepts on reacquisition of motor control and therapist guidelines for rehabilitation technology design. J Neuroeng Rehabil 2009; 6: 1 .

10 Kirshblum SC, Biering-Sorensen F, Betz R, Burns S, Donovan W, Graves DE et al. International Standards for Neurological Classification of Spinal Cord Injury: cases with classification challenges. J Spinal Cord Med 2014; 37: 120-127.

11 Timmermans AAA, Geers RPJ, Franck JA, Dobbelsteijn P, Spooren AIF, Kingma $\mathrm{H}$ et al T-TOAT: a method of task-oriented arm training for stroke patients suitable for implementation of exercises in rehabilitation technology. In: Timmermans AAA, Geers RPJ, Franck JA, Dobbelsteijn P, Spooren AIF, Kingma $\mathrm{H}$ et al. (eds). IEEE 11th International Conference on Rehabilitation Robotics. Kyoto International Conference Center: Japan, 2009

12 Spooren AI, Janssen-Potten YJ, Kerckhofs E, Bongers HM, Seelen HA. Evaluation of a task-oriented client-centered upper extremity skilled performance training module in persons with tetraplegia. Spinal Cord 2011; 49: 1049-1054.

13 Lund AM. Measuring usability with the USE questionnaire 2001 (accessed on 4 september 2014). Available from: http://www.stcsig.org/usability/newsletter/0110_measuring_with_use.html.

14 Markland D, Hardy L. On the factorial and construct validity of the Intrinsic Motivation Inventory: conceptual and operational concerns. Res Q Exerc Sport 1997 68: 20-32. 
15 Devilly GJ, Borkovec TD. Psychometric properties of the credibility/expectancy questionnaire. J Behav Ther Exp Psychiatry 2000; 31: 73-86.

16 Post MW, Van Lieshout G, Seelen HA, Snoek GJ, Ijzerman MJ, Pons C. Measurement properties of the short version of the Van Lieshout test for arm/hand function of persons with tetraplegia after spinal cord injury. Spinal Cord 2006; 44: 763-771.

17 Spooren Al, Janssen-Potten YJ, Post MW, Kerckhofs E, Nene A, Seelen HA Measuring change in arm hand skilled performance in persons with a cervical spinal cord injury: responsiveness of the Van Lieshout Test. Spinal Cord 2006; 44: 772-779.
18 Bluvshtein V, Front L, Itzkovich M, Aidinoff E, Gelernter I, Hart J et al. SCIM III is reliable and valid in a separate analysis for traumatic spinal cord lesions. Spinal Cord 2011; 49: 292-296.

19 Mulcahey MJ, Hutchinson D, Kozin S. Assessment of upper limb in tetraplegia: considerations in evaluation and outcomes research. J Rehabil Res Dev 2007; 44: 91-102.

20 Haisma JA, Bussmann JB, Stam HJ, Sluis TA, Bergen MP, Dallmeijer AJ et al. Changes in physical capacity during and after inpatient rehabilitation in subjects with a spinal cord injury. Arch Phys Med Rehabil 2006; 87: 741-748. 